CAPÍTULO 7

\title{
Microextração em fase sólida para obtenção de compostos voláteis em maçãs e derivados
}

Jossiê Zamperetti Donadel, Valdecir Carlos Ferri, Roger Wagner, Tassiane dos Santos Ferrão

https://doi.org/10.4322/mp.978-65-991393-3-8.c7

\section{Resumo}

A atenção de pesquisadores em relação aos atributos sensoriais de maçãs e derivados tem sido crescente com 0 intuito de obter maior aceitação pelos consumidores. Atributos como sabor, odor e aroma provenientes do perfil de compostos voláteis (CV) estão envolvidos e determinam a qualidade tanto da fruta in natura, quanto de seus derivados, como o suco. Técnicas analíticas como a destilação e extração com solventes, podem ocasionar degradação térmica e extração simultânea de contaminantes, prejudicando a acurácia da análise de CV de frutas, logo, o emprego de uma técnica de análise menos onerosa e limpa se faz necessário. Os objetivos deste trabalho foram fazer uma revisão da literatura sobre a microextração em fase sólida do headspace combinada à análise por cromatografia em fase gasosa (HS-SPME/GC-MS) ressaltando suas vantagens e apresentar as aplicações práticas dessa técnica e impactos na qualidade da maçã e seus derivados pós-colheita. Este artigo demonstrou que a HS-SPME é rápida, precisa, facilmente empregada e mais adequada, tanto do ponto de vista ambiental quanto econômico para a análise dos biomarcadores voláteis característicos de odor e aroma de maçãs e derivados.

Palavras-chave: armazenamento, aroma, atributos de qualidade.

\section{Introdução}

Os alimentos são constituídos por inúmeras substâncias químicas inorgânicas e orgânicas não voláteis, como água, sais minerais, cinzas, lipídeos, proteínas, carboidratos, entre outros compostos. Por outro lado, a constituição química relacionada ao odor e aroma dos alimentos é composta por substâncias voláteis ou semivoláteis (RISTICEVIC et al., 2009). Conforme mencionado por Bangerth, Song e Streif (2012), os voláteis do aroma são um atributo importante do sabor em quase todas as frutas das árvores. Esses autores mencionam que fisiologistas e bioquímicos foram os primeiros interessados nos voláteis do aroma há um século (POWER; CHESTNUT, 1920), porém estavam mais preocupados com a composição e estrutura química desses compostos do que com seu impacto no sabor da fruta e na percepção do consumidor (BANGERTH; SONG; STREIF, 2012). De acordo com esses autores, o mercado atacadista e varejista e até mesmo os horticultores só recentemente descobriram os voláteis e os aromas como índices de qualidade importantes para a satisfazer as demandas dos consumidores e/ou promover as vendas. 
Somente com o advento da cromatografia gasosa (GC), na segunda metade do século $\mathrm{XX}$, foi possível obter avanços significativos na análise da fração volátil. Um progresso real referente à composição química e às vias biossintéticas dos voláteis do aroma foi alcançado somente nas décadas de 60 e 70 (DRAWERT et al., 1968; DRAWERT, 1975; PAILLARD, 1979). Em meados do século XXI, foi estabelecido por Fellman et al. (2000) que o metabolismo de ácidos graxos e aminoácidos ramificados podem servir como precursores para a biossíntese de voláteis de aroma em frutas.

Com a ajuda de GC combinada com a espectrometria de massa, foi possível identificar centenas de compostos voláteis. Além disso, o estabelecimento de "limiares olfativos" proporcionou a oportunidade de categorizar esses compostos de acordo com sua importância sensorial. Isso reduziu o número de compostos considerados importantes sensorialmente, mas aumentou as demandas por instrumentação sensível e de alta resolução porque os limiares olfativos e as taxas de produção de alguns desses compostos pareciam ser muito baixos (BANGERTH; SONG; STREIF, 2012). Em maçãs, por exemplo, mais de 300 compostos voláteis foram identificados e destes, apenas 30 a 40 são considerados como compostos de impacto de sabor (CUNNINGHAM et al., 1986). Nessas frutas, o impacto de vários tratamentos pré e pós-colheita nas taxas de produção da maioria desses compostos voláteis é afetado em um grau semelhante, sugerindo-se que muitos compostos voláteis são derivados de vias bioquímicas semelhantes (BANGERTH; SONG; STREIF, 2012). No entanto, Brackmann, Streif e Bangerth (1993) reportaram que a produção de éster ramificado e não ramificado em maçã armazenada com oxigênio ultrabaixo (ULO) foi diferentemente afetada por diferentes pressões parciais de $\mathrm{CO}_{2}$ na atmosfera de armazenamento. Desse modo, com o emprego de metodologias experimentais, foi possível obter um conhecimento mais aprofundado sobre os efeitos dos procedimentos na produção, armazenamento e manuseio relacionados à emissão de aromas voláteis em várias frutas (BANGERTH; SONG; STREIF, 2012).

Em relação à análise dos compostos presentes nas frutas, procedimentos para isolamento objetivando ao menos sua identificação, além da sua quantificação, foram estabelecidos para aproveitar sua volatilidade. Muitas vezes, as técnicas convencionais de preparo de amostras para que a análise seja representativa de aroma e sabor são morosas ou apresentam modificações no perfil dos compostos (RISTICEVIC et al., 2009). Além disso, grandes quantidades de amostras e uso de solventes orgânicos tóxicos são requeridas nesses métodos convencionais de amostragem (KATAOKA; LORD; PAWLISZYN, 2000).

Técnicas convencionais de isolamento de compostos voláteis como a destilação e extração com solventes, embora sejam simples, possuem desvantagens como a degradação térmica e extração simultânea de contaminantes (PARLIMENT, 1997). Outros estudos reportaram a comparação entre os métodos de extração com solvente e análise do headspace e indicaram o uso da análise do headspace, uma vez que possibilitou a extração de ésteres com notas frutais, portanto, sendo o método mais indicado para análise de compostos voláteis em frutas (KAFKAS et al., 2005; VARMING et al., 2004). Nesse sentido, a abordagem que envolve a análise de headspace estático e dinâmico tem sido utilizada com maior frequência, por propiciar uma representação mais fidedigna da percepção do olfato (FRANCO; JANZANTTI, 2004). Portanto, o isolamento dos compostos voláteis é uma etapa muito importante a qual merece atenção, uma vez que as impurezas dos solventes podem ser transferidas para as amostras e causar interferências nas análises ou a matriz pode sofrer algum tipo degradação. Nesse sentido, para analisar esses constituintes de alimentos, técnicas mais limpas e com pouco uso de solvente se fazem necessárias, a técnica de microextração em fase sólida no headspace confinado da amostra (HS- 
SPME-do inglês Headspace Solid Phase Microextraction), ou simplesmente SPME se apresenta como alternativa mais verde e com menores interferentes sendo carregados para as matrizes.

A HS-SPME está se difundindo mundialmente e inúmeros pesquisadores já utilizam para análise de compostos voláteis conduzindo para várias finalidades, atribuindo algumas modificações na amostragem dependendo do tipo de matriz a ser analisada, sendo aplicada para apenas identificar o perfil volátil, diferenciar origem geográfica (GIANNETTI et al., 2017, MEDINA et al., 2019), bem como avaliar a influência do tipo de armazenamento na fração volátil de frutas (BOTH et al., 2017; DONADEL et al., 2019; QIN; PETERSON; BRADIE, 2018; THEWES et al., 2017a, b).

O aroma e o odor emitidos pelas frutas e seus produtos derivados são atributos importantes da qualidade, em decorrência da presença de substâncias de diversas classes químicas, como álcoois, aldeídos, ésteres, ácidos carboxílicos e cetonas. Nesse sentido, o estudo do perfil de compostos voláteis das frutas desempenha um papel relevante e norteador em relação à avaliação da qualidade dos alimentos tanto na produção, quanto no armazenamento, comercialização e consumo. Logo, os objetivos deste trabalho foram: i) fazer uma revisão da literatura sobre a microextração em fase sólida, pois é uma técnica analítica limpa, verde, destinada à análise de compostos voláteis de maçã e seus derivados e ii) apresentar as aplicações práticas dessa técnica e impactos na qualidade da maçã e seus derivados pós-colheita. Portanto, a SPME pode ser aplicada com sucesso na análise de compostos voláteis que caracterizam as amostras de frutas, sucos e bebidas de frutas.

\section{Fonte de dados e estratégia de pesquisa}

Uma revisão abrangente foi conduzida pesquisando sistematicamente na base de dados ScienceDirect da editora Elsevier para todos os estudos relacionados aos compostos voláteis envolvendo a técnica de microextração em fase sólida relacionada com voláteis de maçãs até 03 de agosto de 2020. Títulos de assuntos relacionados ao objeto de estudo (volatile compounds of apple by SPME) e outros termos-chave (aroma, apple) foram considerados para pesquisas científicas, que empregaram a microextração em fase sólida para análises tanto qualitativas como quantitativas de compostos voláteis presentes em maçãs e seus derivados em diversos países do mundo. Os critérios de exclusão foram artigos que não atenderam aos critérios de inclusão mencionados acima. Para a redação desta revisão foi selecionado um total de 80 referências que apresentavam as informações e dados de interesse dos autores.

\section{A importância da maçã}

A maçã (Malus domestica Borkh) é um dos vegetais mais produzidos e consumidos mundialmente. Sua produção é uma importante atividade econômica, uma vez que está fruta é produzida em escala global sendo majoritariamente consumida in natura, sendo que 7 milhões de toneladas foram desperdiçadas em 2016 (HEUZÉ et al., 2020). Somente no ano de 2017, mais de 80 milhões de toneladas de maçã foram produzidas. Dentre estas, a produção no Brasil alcançou mais de 1 milhão de toneladas na safra 2017/2018 (KIST et al., 2019) e as cultivares 'Gala' e a 'Fuji' e suas mutantes destacam-se no estado do Rio Grande do Sul, culminando em mais de $90 \%$ da safra 2019/2020 (AGAPOMI, 2020).

O consumo da maçã pode trazer vários benefícios à saúde humana. Em estudos observacionais, a ingestão mais alta de maçã está associada ao menor risco de doença coronariana e derrame (LARSSON; VIRTAMO; WOLK, 2013), além de efeitos 
benéficos na função vascular e pressão arterial (BONDONNO et al., 2012), de lipídios (JENSEN et al., 2009), inflamação (CHUN et al., 2008) e potencial atividade antihiperglicêmica por inibição de enzimas digestivas do amido, incluindo $\alpha$-amilase e $\alpha$ glucosidase in vitro e in vivo (LI et al., 2019). Além disso, as maçãs possuem polifenóis, como di-hidrochalconas, flavonóis, hidroxicinamatos e fibras, que incluem celulose, hemicelulose e pectina (VALERIO et al., 2020). Pesquisadores também reportam que a fruta pode ser um transportador adequado para células probióticas, devido às suas propriedades nutricionais e funcionais (EMSER et al., 2017). Estudos in vitro demonstraram o efeito benéfico dos componentes da maçã na composição da microbiota intestinal e no padrão metabólico (KOUTSOS et al., 2017). Esses mesmos autores demonstraram que polissacarídeos não digeríveis e polifenóis que atingem 0 cólon agiram como substratos para a fermentação colônica, produzindo ácidos fenólicos e ácidos graxos de cadeia curta, cujos benefícios à saúde do consumidor já são conhecidos.

Os dados estatísticos dos órgãos como a Food and Agriculture Organization (FAO) revelam uma elevada produção de maçãs no mundo todo. Em decorrência disso, armazená-las de forma adequada tanto em armazenamento refrigerado (AR) quanto em atmosfera controlada (AC) torna-se essencial para reduzir perdas pós-colheita, além de regular a oferta da fruta no período da entressafra no comércio local e internacional.

No armazenamento refrigerado há controle da umidade relativa e as maçãs são armazenadas em baixa temperatura, sendo considerada o principal fator responsável pela supressão do metabolismo dos frutos (EKMAN; GOLDING; McGLASSON, 2005). Por outro lado, a conservação é observada apenas em um período restrito de poucos meses, em que há reduções significativas na suculência e na firmeza. Além dessas alterações, aumento de danos por degenerescência e formação de polpa farinácea podem ser evidenciados, quando esse tempo de armazenamento é ultrapassado (SAQUET; BRACKMANN; STORC, 1997). Já quando se armazena em atmosfera controlada (AC), além do controle da umidade relativa e da temperatura, pode-se alterar a concentração de gases $\left(\mathrm{O}_{2}\right.$ e $\left.\mathrm{CO}_{2}\right)$ dentro das câmaras de armazenamento (BRACKMANN et al., 2008; LUMPKIN et al., 2015), propiciando a estocagem por um maior período.

No Brasil, o armazenamento das maçãs em AC pode se estender até 240-270 dias (BRACKMANN et al., 2013; WEBER et al., 2013). Entretanto, embora as pressões parciais possam ser controladas, ainda pode ocorrer a redução de compostos de aroma desejáveis presentes nas maçãs armazenadas, além de distúrbios fisiológicos (BRACKMANN; STREIF; BANGERTH, 1993; ECHEVERRÍA et al., 2004; MATHEIS et al., 2005), pois em AC não é possível monitorar o metabolismo do fruto em tempo real. Se as concentrações gasosas de AC utilizadas durante o armazenamento forem inadequadas para uma determinada cultivar (LUMPKIN et al., 2015), além da ocorrência de degenerescência de polpa (WEBER et al., 2013), pode haver indução do metabolismo fermentativo, com consequente acúmulo de acetaldeído, etanol e acetato de etila. Caso essas substâncias sejam encontradas em concentrações muito altas, tornam-se compostos de sabor indesejáveis (off flavor), com a possibilidade de causar distúrbios fisiológicos (SAQUET; STREIF, 2008; WRIGHT; ARUL; PRANGE, 2015).

Nos últimos anos, outras alternativas de armazenagem têm sido exploradas, como as que empregam a atmosfera controlada dinâmica (ACD), onde a pressão parcial de $\mathrm{O}_{2}$ varia ao longo do período de armazenamento (PRANGE et al., 2005) de acordo com o limite mínimo de oxigênio (do inglês low limit oxygen - LOL) tolerado pelo fruto, diferentemente da $A C$, onde a $\mathrm{pO}_{2}$ permanece estática. Segundo Prange (2018), três 
métodos têm sido empregados para detectar o LOL em ACD, incluindo o monitoramento do $\mathrm{O}_{2}$ durante o armazenamento pela produção de etanol (VELTMAN; VERSCHOOR; RUIJSCH VAN DUGTEREN, 2003), monitoramento pela emissão de fluorescência de clorofila (WRIGHT et al., 2015) e mais recentemente o monitoramento pelo quociente respiratório (WEBER et al., 2017). Dessa forma, o armazenamento em ACD tem sido uma alternativa que proporciona melhor qualidade físico-química e sobretudo promove a maior emissão de compostos voláteis característicos de qualidade de odor e aroma da maçã (BOTH et al., 2017, DONADEL et al., 2019; THEWES et al., 2017a).

\subsection{Compostos voláteis em maçãs: importância e biossíntese}

Os compostos voláteis (CV) constituem os atributos de qualidade de sabor, odor e aroma. À medida que ocorre o amadurecimento dos frutos, esses compostos mudam em resposta à maturidade e às condições de armazenamento (BANGERTH; SONG; STREIF, 2012) e, consequentemente, são atributos sensoriais relevantes para a aceitação das maçãs pelos consumidores (LÓPEZ et al., 2007).

Sensações complexas relacionadas com o gosto, com o aroma e diversas sensações bucais conferem o sabor específico das maçãs (BALDWIN, 2002; PÉREZ; SANS, 2008). Os açúcares e ácidos orgânicos, por sua vez, designam os gostos predominantes. Já uma mistura complexa de muitos compostos voláteis percebidos sensorialmente através da via retronasal conferem o aroma característico da fruta (NOGUEROL-PATO et al., 2012; SALAZAR; OROSCO, 2011) e sua composição pode variar conforme a espécie, variedade e estágio de maturação (CUNNINGHAM et al., 1986; PÉREZ; SANS, 2008; SANS; OLÍAS; PÉREZ, 1997).

A literatura científica reporta que já foram detectados mais de trezentos compostos voláteis em maçãs (FORNEY; MATTHEIS; BALDWIN, 2009). Os compostos predominantes são álcoois, aldeídos, cetonas, terpenoides, sesquiterpenos, éteres e principalmente ésteres (BOTH et al. 2014; DONADEL et al., 2019; ROWAN et al., 1999; THEWES et al., 2017a, b) e os de maior impacto são os álcoois e ésteres (DIXON; HEWETT, 2001). É válido ressaltar que a contribuição relativa de cada um desses compostos voláteis pode ser determinada pela unidade de odor, que consiste na razão entre a concentração de um composto e seu limiar de percepção olfativo (TAKEOKA; BUTTERY; FLASH, 1992), sendo que dentre os compostos voláteis emitidos pelas maçãs, os ésteres têm um grande impacto no aroma.

Os compostos voláteis são biossintetizados por meio do envolvimento de várias enzimas, como lipoxigenase (LOX), álcool desidrogenase (ADH), álcool aciltransferase (AAT) e substratos como carboidratos, proteínas e principalmente lipídios (SALAZAR; OROSCO, 2011). Vários autores reportam que as substâncias voláteis características das maçãs são formadas a partir de ácidos graxos insaturados, destacando-se no período climatérico os ácidos oleico, linoleico e linolênico (DI, 2005; GALLIARD, 1968; MEIGH; HULME, 1965; MEIGH; JONES; HULME, 1967; NIE; SUN; SONG; BANGERTH, 2003). De acordo com Fellman et al. (2000), esses precursores de compostos voláteis são liberados por meio da atividade enzimática de lipases que atuam nos lipídios da membrana celular e são catabolizados através de duas rotas oxidativas: $\beta$-oxidação, mais importante em frutas íntegras e via da lipoxigenase (LOX), predominante quando ocorre ruptura dos tecidos vegetais (PÉREZ; SANZ, 2008; SANZ; OLÍAS; PÉREZ, 1997).

A $\beta$-oxidação ocorre quando os ácidos graxos sofrem uma ativação, podendo atravessar a membrana mitocondrial interna e chegar na matriz mitocondrial. Nesse 
processo oxidativo atuam várias enzimas, ocorrendo em quatro passos, resumidos da seguinte forma: oxidação de Acil-CoA, transformando em trans-2-enoil-CoA, utilizando flavina-adenina-dinucleotídeo (FAD) como cofator e $\mathrm{O}_{2}$, como aceptor de elétrons, seguida de hidratação, formando 3-hidroxiacil-CoA. O terceiro passo consiste na desidrogenação, requerendo nicotinamida-adenina-dinucleotídeo (NAD) como cofator, formando 3-cetoacil-CoA, então segue-se a tiólise, ou seja, a quebra da extremidade tiol de 3-cetoacil-CoA, resultando em uma molécula de acetil-CoA e uma de acil-CoA, a qual retorna ao ciclo de $\beta$-oxidação (BAKER et al., 2006; ESPINO-DÍAS et al., 2016; GOEPFERT et al., 2005; GOEPFERT; POIRIER, 2007; GRAHAM; EASTMOND, 2002; MATHEWS et al., 2002). Esta molécula de Acil-CoA liga-se a um álcool para formar os ésteres pela ação da enzima álcool aciltransferase (AAT) (SANZ; OLÍAS; PÉREZ, 1997). A outra via oxidativa refere-se à rota da LOX, cuja ativação se dá em decorrência da ruptura dos tecidos vegetais, tendo como produtos substâncias voláteis de 6 a 9 carbonos (SANZ; OLÍAS; PÉREZ, 1997). A LOX age principalmente sobre os ácidos graxos insaturados linoleico (C18:2) e linolênico (C18:3) (PÉREZ; SANZ, 2008), os quais são liberados a partir de triacilgliceróis, fosfolípidos e glicolípidos pela ação de acil hidrolases (REINECCIUS, 2006). Logo, a formação de metabólitos voláteis como álcoois, aldeídos, ácidos e ésteres é o resultado da degradação desses ácidos graxos por processo oxidativo (PÉREZ; SANZ, 2008). Outra fonte expressiva de compostos voláteis que conferem o aroma de frutas e vegetais refere-se ao metabolismo dos aminoácidos (ESPINO-DÍAZ et al., 2016). Nesse caso, enzimas promovem transformações dessas moléculas precursoras e formam produtos como aldeídos, álcoois, ácidos e principalmente ésteres de cadeia ramificada (WYLLIE; FELLMAN, 2000).

Pesquisas demonstraram que maçãs do grupo 'Gala', bem como 'Galaxy', apresentam altas concentrações de acetato de butila, acetato de 2-metilbutila e acetato de hexila, que são os ésteres mais importantes desse grupo de cultivares (BOTH et al., 2014; SALAZAR; OROZCO, 2011). Já para a maçã 'Granny Smith', acetato de etila, butanoato de etila, acetato de butila, propanoato de etila, 2-metil butanoato de etila e 2-metil butanoato de propila foram os principais ésteres responsáveis pelo aroma de amostras de suco de maçã (NIU et al., 2019), os compostos 1-butanol e butanoato de butila, podem ser utilizados como indicadores de qualidade na avaliação da qualidade da maçã 'Fuji' (LEE; JEONG; JANG, 2017). Portanto, a produção de substâncias voláteis em maçãs é o resultado de uma combinação de rotas metabólicas em que diversos processos fisiológicos e mecanismos de controle estão envolvidos, em que a presença desses compostos é influenciada por fatores genéticos, práticas culturais, maturação da safra e condições de armazenamento (ESPINO-DÍAZ et al., 2016).

\section{Histórico, princípios e vantagens da técnica de microextração em fase sólida (SPME)}

A microextração em fase sólida foi introduzida pela primeira vez por Arthur e Pawliszyn em 1990. A SPME é uma técnica avançada de pré-tratamento de amostras que integra amostragem, extração, concentração e introdução de amostras em uma única etapa sem solvente (ARTHUR; PAWLISZYN, 1990). Baseia-se no mecanismo físico-químico da partição do analito entre a fase de extração imobilizada em uma fibra de sílica fundida e a matriz. Após o equilíbrio ser alcançado ou um tempo bem definido, os compostos sorvidos na fibra podem sofrer dessorção térmica, quando se expõe a fibra no injetor de um cromatógrafo a gás (GC) (XU et al., 2016). Fatores como a fácil miniaturização, a automação de dispositivos, além da possibilidade de acoplamento com instrumentos cromatográficos destacam-se como as principais vantagens da SPME (XU et al., 2016). Atualmente, esse tipo específico de extração de compostos voláteis é amplamente aceito e com constante crescimento de novas pesquisas na 
área de alimentos, com vastas publicações na literatura científica, o que ressalta sua importância para a análise de alimentos.

Os parâmetros que determinam a eficiência da extração devem ser otimizados para que haja a alta qualidade nas análises baseadas na técnica de SPME. Dentre esses parâmetros é possível investigar a química da fase de extração, modo de extração, método de agitação, modificação da amostra $(\mathrm{pH}$, força iônica, conteúdo de solvente orgânico), temperatura da amostra, tempo de extração e condições de dessorção (RISTICEVIC et al., 2009). Ainda dentro desses parâmetros, o revestimento de fibra é um dos fatores mais críticos que influenciam o desempenho dos métodos de SPME. É importante ressaltar que a polaridade do revestimento e sua seletividade em relação aos analitos de interesse, em contraste com outro componente da matriz, determina qual deve ser o revestimento de fibra adequado para um composto específico de interesse.

Xu et al. (2013) reportaram resumidamente os novos materiais de revestimento SPME. Dentre todos os materiais de revestimento avaliados experimentalmente, 0 polidimetisiloxano (PDMS), como revestimento líquido com superfície lisa e homogênea, sofre menos com o efeito irreversível de incrustação causado pelos componentes da matriz do que com revestimentos sólidos (BELTRAN; LÓPEZ; HERNÁNDEZ, 2000). Contudo, era pouco sensível para extrair alguns compostos de interesse. Desse modo, para criar um novo tipo de revestimento de fibra SPME, uma modificação dos revestimentos comerciais de fibra SPME existentes foi realizada, alcançando compatibilidade com a matriz. A fibra com revestimento de carboxen/divinilbenzeno/polidimentilsiloxano (Car/DVB/PDMS) possuiu maior eficiência e permitiu maior número de extrações com a mesma fibra, além de manter a sensibilidade do revestimento original para os analitos de interesse, quando submetida diretamente a uma matriz complexa (PAWLISZYN, 2009). Logo, essa fibra acabou sendo a mais frequentemente utilizada para análise de alimentos (XU et al., 2016).

\section{A HS-SPME aplicada na análise de maçãs}

\subsection{SPME na análise de maçãs e derivados}

Alimentos são matrizes muito complexas, logo um método adequado de preparo de amostra é uma grande preocupação dos analistas. A literatura reporta a avaliação de compostos voláteis em maçãs bem como em seus derivados, principalmente em bebidas. A técnica de HS-SPME-GC/MS foi empregada para extração e caracterização de compostos orgânicos voláteis no suco de maçã. Para essa análise, uma fibra PDMS de $65 \mu \mathrm{m}$ foi usada para SPME e mais de setenta compostos voláteis foram determinados a partir de diferentes classes químicas (SCHMUTZER et al., 2014).

Alguns estudos que empregam a análise por HS-SPME ressaltam a composição volátil como variável crucial para a caracterização entre diferentes cultivares e seus produtos derivados de acordo com as origens geográficas. Foi possível distinguir duas diferentes regiões no nordeste da Itália: Alto Ádige-Tirol do Sul (AA-ST) e Friuli Venezia Giulia (FVG), pela análise de compostos voláteis de quarenta e duas cultivares de maçãs encontradas nessa região (GIANNETTI et al., 2017). Em particular, a elaboração de perfis cromatográficos da fração de sabor, após prétratamentos adequados de sinal e aplicando ferramentas quimiométricas resultou em modelos estatísticos com alta precisão e boa capacidade de classificação que permitiram discriminar as maçãs cultivadas em FVG das provenientes de AA-ST, bem como grupos de amostras de AA-ST sendo cultivados sob diferentes práticas agrícolas (método convencional ou orgânico). Além disso, os modelos estatísticos empregados 
evidenciaram que a informação discriminante estava ligada aos compostos voláteis mais característicos (alguns ésteres, álcoois e terpenos) presentes na polpa de maçãs para as categorias de amostras consideradas (GIANNETTI et al., 2017).

Através da microextração em fase sólida do headspace combinada com a cromatografia em fase gasosa e espectrometria de massas (HS-SPME-GC/MS) em conjunto com ferramentas quimiométricas, Medina et al. (2019) estabeleceram características distintas para a discriminação de sucos de maçã de variedades regionais (Rijo, Verde, Ribeiro e Azedo) e origem geográfica (Prazeres e Santo da Serra), da llha da Madeira, em Portugal.

Medina et al. (2019) revelaram que é possível detectar compostos voláteis de sabor que estão presentes em concentrações diminutas em matrizes complexas e que podem ser responsáveis pela discriminação entre sucos de maçãs cultivadas nas diferentes regiões geográficas estudadas. Os resultados obtidos revelaram uma perfeita discriminação entre as diferentes variedades de maçã, com sucos de maçã 'Rijo' como amostras com maior concentração relativa de etanol, butanoato de etila, 2metilbutanoato de etila e hexanoato de etila. Além disso, este estudo permitiu uma classificação geográfica de sucos de maçã 'Azedo', com base na origem, destacando como características discriminatórias acetato de etila, 2-metil-1-propanol, hexanoato de etila e tolueno (descritos pela primeira vez em sucos de maçã).

Alguns estudos destacam os ésteres eficientemente relacionados à qualidade do suco de maçã 'Fuji', 'Red Delicious' e 'Granny Smith' (NIU et al., 2019). Esses autores reportaram a identificação e quantificação de 20 ésteres dessas três cultivares, empregando a técnica de HS-SPME combinada com cromatografia em fase gasosa com olfatometria (GC-O). Do total de ésteres encontrados, seis deles foram selecionados como os principais compostos ativos de odor. Além disso, acetato de etila, butanoato de etila, acetato de butila, propanoato de etila, 2-metil butanoato de etila e 2-metil butanoato de propila foram os principais ésteres para o aroma da amostra de suco de maçã 'Granny Smith' (NIU et al., 2019). Ademais, foi reportado que o efeito de adição ou efeito sinérgico ocorreu possivelmente quando os ésteres tinham estrutura e aroma semelhantes. No entanto, isso não significava que todos os compostos com estrutura e aroma semelhantes pudessem apresentar um efeito sinérgico após a mistura. Notadamente os compostos butanoato de etila e acetato de butila tiveram um efeito sinérgico (NIU et al., 2019). A fim de obter uma melhor compreensão da mistura desses dois compostos, uma avaliação sensorial e análise por nariz eletrônico foram implementadas para investigar as características do perfil de aroma das amostras de ésteres e suas variações de sabor. Foi observada diferença significativa nas notas frutadas, azedas e verdes entre os ésteres não misturados e a mistura binária (NIU et al., 2019).

Em estudos realizados por Kebede et al. (2020) marcadores em potencial para mudanças voláteis durante a vida útil em ambiente e acelerada do suco de maçã pasteurizado foram revelados. As mudanças voláteis foram monitoradas a 20, 30 e 40 ${ }^{\circ} \mathrm{C}$ usando um método de impressão digital aplicando HS-SPME/GC-MS. Utilizando quimiometria moderna e seleção de características, hexanal, trans-2-hexenal, sulfeto de dimetil, furfural, acetato de etila e 1-pentanol foram escolhidos como potenciais marcadores de vida em prateleira. Os voláteis associados ao aroma de maçã verde, gramínea e fresca, como hexanal e trans-2-hexenal, diminuíram durante 0 armazenamento, enquanto a carga térmica e os compostos associados ao escurecimento, como sulfeto de dimetila e furfural, aumentaram durante 0 armazenamento. Hexanal e trans-2-hexenal podem ser marcadores para monitorar a alteração de caráter semelhante à maçã verde. O furfural e o sulfureto de dimetil 
podem ser marcadores de abuso de temperatura durante o processamento e o armazenamento do suco. E furfural também pode ser um indicador para 0 escurecimento do suco (KEBEDE et al., 2020).

Perestelo et al. (2019) focaram seus estudos na diferenciação de sucos de frutas frescas e processadas utilizando composição volátil. Esses autores estabeleceram que a atenção especial deve ser dada aos sucos de frutas processados para evitar os possíveis efeitos deletérios associados à formação de compostos furânicos (por exemplo, tratamento térmico), uma vez que seu conteúdo furânico foi significativamente maior em comparação ao dos sucos de frutas frescas. O conhecimento obtido neste trabalho permite a introdução de modificações no processo envolvido no processamento de sucos, para que se melhore as características sensoriais dos sucos processados, contribuindo para uma melhor aceitação pelos consumidores.

Outros estudos reportam a relação entre compostos voláteis com a percepção de doçura da maçã (APREA et al., 2017). Quarenta lotes de maçãs, pertencentes a 17 diferentes cultivares, foram coletados e submetidas a análise. Treze compostos foram positivamente correlacionados com doçura de um total de dezesseis significativos relatados $(p<0,05)$, sendo que o principal grupo de compostos foram os ésteres ( 7 dos 16 compostos). Os ésteres de propila apresentaram os maiores coeficientes de correlação positivos para a percepção de doçura, como o 2-metilbutanoato de propila, hexanoato de propila e propanoato de propila, respectivamente, já os compostos que foram negativamente correlacionados foram 1-octen-3-ona, 2-heptenal e 1-octen-3-ol. (APREA et al., 2017). Diante do que foi abordado, a SPME combinada à análise por GC/MS associada a tratamentos estatísticos adequados, pode ser vista como uma metodologia analítica de extração de compostos voláteis versátil, possibilitando assistir o monitoramento de marcadores voláteis de inúmeras cultivares. A presença desses compostos, que mesmo em pequenas quantidades, servem como indicadores de qualidade em pesquisas relacionadas à origem geográfica e de biomarcadores de sabor, odor e aroma.

\subsection{SPME aplicada ao armazenamento de maçãs}

No armazenamento de maçãs, concentrações de $\mathrm{O}_{2}$ e $\mathrm{CO}_{2}$ na atmosfera de armazenamento podem ocasionar alterações metabólicas na fruta e podem refletir na qualidade pós-colheita da maçã. Nesse sentido, compostos voláteis biomarcadores de odor e qualidade de maçãs das variedades 'Royal Gala', 'Fuji Kiku', 'Galaxy' e 'Fuji Suprema' foram reportados por Both et al. (2017), Donadel et al. (2019) e Thewes et al. (2017a, b), respectivamente, com destaque para a classe dos ésteres de acetato e ésteres etílicos.

Nesses estudos foram utilizados diferentes tipos de armazenamentos (atmosfera controlada convencional, comparada com atmosfera controlada dinâmica monitorada por fluorescência de clorofila e monitorada pelo quociente respiratório com pressões parciais extremamente baixas de oxigênio), onde os ésteres foram os compostos marcadores de odor e aroma mais importantes para as variedades de maçãs. Notadamente, os compostos voláteis mais abundantes e importantes emitidos para o odor e aroma são os ésteres. Além disso, uma avaliação metabólica comparativa de maçãs 'Granny Smith' e 'Red Delicious' foi abordada para explicar processos comuns e divergentes quando essas frutas fossem submetidas a condições de armazenamento com baixos níveis de oxigênio (Ultra Baixo oxigênio, ULO, a 0,9 kPa de oxigênio e ACD-CF, entre 0,2 e $0,55 \mathrm{kPa}$ de $\mathrm{O}_{2}$ ) ao longo de 6 meses de armazenamento. Foi possível elucidar que, particularmente, ésteres etílicos foram 
produzidos mais abundantemente por maçãs 'Red Delicious' e essa resposta afetou outras características composicionais, (BRIZZOLARA et al., 2017).

Outro estudo focou em compostos indesejáveis, denominados off flavor, incluindo 1butanol e butanoato de butila. Foi evidenciado que a quantidade desses voláteis em maçãs 'Fuji' tratadas com 1-metilciclopropeno (1-MCP) diminuiu ao longo de 5 meses e depois aumentou após 7 meses. Por outro lado, nas maçãs que não foram tratadas com o fitorregulador, não houve alterações significativas desses analitos durante os 7 meses de armazenamento $(p<0,05)$ apresentando também os níveis mais altos desses compostos que as maçãs tratadas com 1-MCP (LEE; JEONG; JANG, 2017). Assim, os autores desse estudo concluíram que 1-butanol e butanoato de butila podem ser indicadores de qualidade na avaliação da qualidade da maçã 'Fuji'.

As alterações nos componentes voláteis das maçãs 'Fuji' foram analisadas em amostras íntegras usando HS-SPME. A qualidade foi avaliada durante 0 armazenamento a longo prazo em temperatura de $4{ }^{\circ} \mathrm{C}$ por 3,6 e 9 meses. A aplicação de álcoois precursores (butanol, hexanol e 2-metilbutanol) resultou em maiores emissões de oito ésteres. Dentre eles, acetato de butila, butanoato de butila, 2-metilbutanotato de butila ou acetato de 2-metilbutil estavam altamente correlacionados com as de seus álcoois precursores (LEE et al., 2017).

Donadel et al. (2019) compararam a produção de compostos voláteis do headspace das câmaras de armazenamento, do headspace dos frutos no shelf life (7 dias a 20 $\left.{ }^{\circ} \mathrm{C}\right)$ e do suco extraído de maçã 'Fuji Kiku' após os 7 dias de shelf life. Esses autores reportaram maiores concentrações dos ésteres etílicos de cadeia ramificada característicos de odor dessa cultivar, como 2-metil propanoato de etila, 3-metil butanoato de etila e 2-metil butanoato de etila em atmosfera controlada dinâmica monitorada por quociente respiratório (ACD-QR), sobressaindo-se daqueles encontrados em atmosfera controlada (AC), devido à indução do metabolismo fermentativo, com maiores emissões de etanol, que serve como precursor para a formação desses ésteres.

Outros estudos identificaram, por meio da HS-SPME, os compostos que contribuem significativamente para o aroma de maçã 'Fuji', sendo eles o acetato de 2-metilbutila, 2-metilpropanoato de etila, 2-metilbutanoato de etila e hexanoato de etila (NIU et al., 2019; THEWES et al., 2017a). Além disso, Thewes et al. (2017a) mostraram, através dessa técnica, que aplicação de 1-MCP suprimiu a produção de compostos voláteis maçãs 'Galaxy', principalmente os ésteres principais após o armazenamento em atmosfera controlada estática e dinâmica.

\section{Considerações finais}

A composição volátil das maçãs e derivados pode ser um indicador de qualidade, tanto de classificação em relação sua origem geográfica, como pode-se estimar o período de armazenamento até a fruta ser lançada ao mercado consumidor sem haver perda dos atributos sensoriais da fruta in natura e seus derivados.

A combinação da técnica de microextração em fase sólida no headspace combinada com a cromatografia em fase gasosa, associada aos métodos de classificação quimiométrica, oferece uma ferramenta valiosa e mais vantajosa, por ser rápida, facilmente aplicada, menos onerosa e confiável para avaliar a rastreabilidade geográfica de diferentes variedades de frutas, além de verificar a autenticidade das frutas provenientes do cultivo orgânico e caracterizá-las em relação aos diferentes tipos de armazenamento. 
A análise por microextração em fase sólida no headspace combinada com a cromatografia em fase gasosa pode ser implementada para a avaliação da qualidade de maçãs, tanto na condição de consumidas in natura como em situações de armazenamento.

O conhecimento sobre os voláteis pode se tornar muito vantajoso economicamente, uma vez que é possível agregar valor ao produto ao estipular sua denominação de origem geográfica baseada no perfil volátil, além de identificar qual melhor tipo de armazenamento pode ser implementado baseado na produção de compostos voláteischave que denotam os odores característicos de diferentes cultivares.

\section{Referências}

AGAPOMI. Associação Gaúcha dos Produtores de Maçã: dados estatísticos - safra 2020. Disponível em: <http://agapomi.com.br/informacoes/dados-estatisticos/>Acesso em: 02 jul 2020.

APREA, E. et al. Sweet taste in apple: the role of sorbitol, individual sugars, organic acids and volatile compounds. Scientific Report. v.7, p. 44950, 2017. https://doi.org/10.1038/srep44950.

ARTHUR, C. L.; PAWLISZYN, P. Solid phase microextraction with thermal desorption using fused silica optical fibers. Analytical Chemistry, v.62, p. 2145-2148, 1990. https://doi.org/10.1021/ac00218a019.

ASSOCIAÇÃO PORTUGUESA DE NUTRIÇÃO. Colher saber. E-book no 45. Porto: Associação Portuguesa de Nutrição; 2017. Disponível em: <https://www.apn.org.pt/documentos/ebooks/AW_EBook_ColherSaber.pdf>. Acesso em: 12 dez. 2020.

ANVISA, 1969. Decreto-Lei $N^{\circ}$ 986, de 21 de outubro de 1969. Institui normas básicas sobre alimentos. Disponível em: <http://www.planalto.gov.br/ccivil_03/decreto-lei/Del0986.htm>. Acesso em: 02 jul. 2020.

BALDWIN, E. A. Fruit and vegetable flavor. In: GROSS, K.C.; WANG, C.Y.; SALTVEIT, M.A., editors. Handling, transportation and storage of fruits, vegetables, and florist and nursery stock. Washington, DC, USA: United States Department of Agriculture (USDA), Agriculture Handbook; 2002. p. 66.

BANGERTH, F.; SONG, J.; STREIF, J. Physiological impacts of fruit ripening and storage conditions on aroma volatile formation in apple and strawberry fruit: A review. Hort Science, v. 47, p. 4-10, 2012. https://doi.org/10.21273/HORTSCI.47.1.4.

BAKER A. et al. Chewing the fat: $\beta$-oxidation in signalling and development. Trends in Plant Science, v. 11, p. 124-32, 2006. https://doi.org/10.1016/j.tplants.2006.01.005.

BELTRAN. J.; LÓPEZ, F.J.; HERNÁNDEZ, F. Solid-phase microextraction in pesticide residue analysis. Journal of Chromatography A, v. 885, p. 389-404, 2000. https://doi.org/10.1016/s0021-9673(00)00142-4.

BONDONNO, C. P. et al. Flavonoid-rich apples and nitrate-rich spinach augment nitric oxide status and improve endothelial function in healthy men and women: $A$ randomized controlled trial. Free Radical Biology and Medicine, v. 52, n. 1, p. 95102, 2012. https://doi.org/10.1016/j.freeradbiomed.2011.09.028. 
BOTH, V. et al. Effect of storage under extremely low oxygen on the volatile composition of 'Royal Gala' apples. Food Chemistry, v.156, p.50-57, 2014. https://doi.org/10.1016/j.foodchem.2014.01.094.

BOTH et al. Effects of dynamic controlled atmosphere by respiratory quotient on some quality parameters and volatile profile of 'Royal Gala' apple after long-term storage. $\begin{array}{llllll}\text { Food Chemistry, } & \text { v. } 215, \quad \text { p. }\end{array}$ https://doi.org/10.1016/j.foodchem.2016.08.009.

BRACKMANN, A.; STREIF, J.; BANGERTH, F et al. Relationship between a reduced aroma production and lipid metabolism of apples after long-term controlled-atmosphere storage. Journal of the American Society for Horticultural Science, v. 118, p. 243247. 1993. https://doi.org/10.21273/JASHS.118.2.243

BRACKMANN, A. et al. Manutenção da qualidade pós-colheita de maçãs 'Royal Gala' e 'Galaxy' sob armazenamento em atmosfera controlada. Ciência Rural, v.38, n.9, p.2478-2484, 2008. http://dx.doi.org/10.1590/S0103-84782008000900010.

BRACKMANN, A. et al. Efeito do estresse inicial por baixo $\mathrm{O}_{2}$ combinado com 1metilciclopropeno na conservação de maçãs 'Royal Gala' armazenadas com ultrabaixo $\mathrm{O}_{2}$. Semina: Ciência Agrária, v. 34, p. 1185-1194, 2013. http://dx.doi.org/10.5433/1679-0359.2013v34n3p1185-1194.

BRIZZOLARA, S. et al. A metabolomics approach to elucidate apple fruit responses to static and dynamic controlled atmosphere storage. Postharvest Biology and Technology, v.127, p.76-87, 2017. http://dx.doi.org/10.1016/j.postharvbio.2017.01.008.

CHUN, O. K. et al. Serum C-reactive protein concentrations are inversely associated with dietary flavonoid intake in US adults. The Journal of Nutrition, v. 138, n. 4, p. 753-760, 2008. https://doi.org/10.1093/jn/138.4.753.

CUNNINGHAM, D.G. et al. Charm analysis of apple volatiles. Food Chemistry, v.19, p.137-147. 1986. https://doi.org/10.1016/0308-8146(86)90107-X.

DIXON, J.; HEWETT, E. W. Exposure to hypoxia conditions alters volatile concentrations of apple cultivars. Journal of the Science of Food and Agriculture, v.81, p.22-29, 2001. https://doi.org/10.1002/1097-0010(20010101)81:1<22::AIDJSFA769>3.0.CO;2-9.

DONADEL, J. Z. et al. Key volatile compounds of 'Fuji Kiku' apples as affected by the storage conditions and shelf life: Correlation between volatile emission by intact fruit and juice extracted from the fruit. Food Research International, v. 125, p. 108625, 2019. https://doi.org/10.1016/j.foodres.2019.108625.

DRAWERT, F. 1975. Formation des aromes a different stades de l'evolution du fruit; Enzimas intervenientes na formação de células309319 Ulrich R. células Facteurs et Regulation de la Maturação de Frutas Centre National Recherche Scientifique Paris, França.

DRAWERT, F. et al. Über die biogenese von aromastoffen bei Pflanzen und Früchten. III. Gaschromatographische Bestandsaufnahme von Apfel aromastoffen Phytochem. v.7, p. 881 883, 1968. 
ECHEVERRÍA, G. et al. Aroma volatile compounds of 'Fuji' apples in relation of harvest date and cold storage technology: a comparison of two seasons. Postharvest Biology $\begin{array}{llll}\text { and } \quad \text { vechnology, } 2004 . & \text { p.29-44, }\end{array}$ https://doi.org/10.1016/j.postharvbio.2003.09.017.

EKMAN, J.H.; GOLDING, J.B.; McGLASSON, W.B. Innovation in cold storage technologies. Stewart Postharvest Review, v. 1, n. 3, p. 1-14, 2005. https://doi.org/10.2212/spr.2005.3.6.

EMSER, K. et al. Lactobacillus plantarum survival during the osmotic dehydration and storage of probiotic cut apple. Journal of Functional Foods, v.38, p. 519-528, 2017. https://doi.org/10.1016/j.jff.2017.09.021.

ESPINO-DÍAZ et al. Biochemistry of apple aroma: A review. Food Technology and $\begin{array}{lllllll}\text { Biotechnology. } & \text { v. } 54, \quad \text { n. } 4, \quad \text { p. 375-394, } 2016 .\end{array}$ https://doi.org/10.17113/ftb.54.04.16.4248.

FAO, 2016. FAOSTAT. Food and Agriculture Organization of the United Nations, Rome, Italy. Disponível em: <http://www.feedipedia.org/node/21446>. Acesso em: 02 jul.2020

FELLMAN, J. K. et al. Factors that influence biosynthesis of volatile flavor compounds in apple fruits. HortScience, v. 35, p. 1026-1033, 2000. https://doi.org/10.21273/HORTSCI.35.6.1026.

FORNEY C.F. MATTHEIS, J.P.; BALDWIN, A.E. Effects on flavor. In: YAHIA, E. M. (Ed) Modified and controlled atmospheres for the storage transportation, and packaging of horticultural commodities. Boca Ratón: CRC Press, 2009, p.119-159.

FRANCO, M. R. B.; JANZANTTI, N. S. Avanços na metodologia instrumental da pesquisa do sabor. In: FRANCO, M. R. B. Aroma e sabor dos alimentos: temas atuais. São Paulo: Livraria Varela, 2004, p. 17-28.

GALLIARD, T. Aspects of lipid metabolism in higher plants. II. The identification and quantitative analysis of lipids from the pulp of pre-and post-climacteric apples. Phytochemistry, v.7, p. 1915-22, 1968. https://doi.org/10.1016/S00319422(00)90751-9.

GIANNETTI, V. et al. Volatile fraction analysis by HS-SPME/GC-MS and chemometric modeling for traceability of apples cultivated in the Northeast Italy. Food Control, v.78, p.215-221, 2017. https://doi.org/10.1016/j.foodcont.2017.02.036.

GOEPFERT S. et al. Molecular identification and characterization of the Arabidopsis $\Delta 3,5, \Delta 2,4$-dienoyl-coenzyme $A$ isomerase, a peroxisomal enzyme participating in the $\beta$-oxidation cycle of unsaturated fatty acids. Plant Physiology, v.138, p. 1947-1956, 2005. https://doi.org/10.1104/pp.105.064311.

GOEPFERT, S.; POIRIER Y. $\beta$-Oxidation in fatt y acid degradation and beyond. Current Opinion in Plant Biology. v. 10, p. 245-51, 2007. https://doi.org/10.1016/j.pbi.2007.04.007.

GRAHAM I. A.; EASTMOND, P. J. Pathways of straight and branched chain fatty acid catabolism in higher plants. Progress in Lipid Research, v. 41, p. 156-81, 2002. https://doi.org/10.1016/S0163-7827(01)00022-4. 
HEUZÉ V. et al. Apple pomace and culled apples. Feedipedia, a programme by INRA, CIRAD, AFZ and FAO. 2020. Disponível em: <https://feedipedia.org/node/20703>. Acesso em: 03 ago. 2020.

JENSEN, E.N. et al. Mini-review: The effects of apples on plasma cholesterol levels and cardiovascular risk-a review of the evidence. The Journal of Horticultural $\begin{array}{llllll}\text { Science \& Biotechnology, } & \text { v. } & 1, & \text { p. } & 34, & 2009 .\end{array}$ https://doi.org/10.1080/14620316.2009.11512592.

KAFKAS, E. et al. Comparison of methodologies for the identification of aroma compounds in strawberry. Turkish Journal of Agriculture and Forestry. v.29, p.383390, 2005.

KATAOKA, H., LORD, H.L., PAWLISZYN, J. Applications of solid-phase microextraction in food analysis. Journal of Chromatography A, v. 880, p.35-62, 2000. https://doi.org/10.1016/s0021-9673(00)00309-5.

KEBEDE, B. et al. Volatile changes during storage of shelf Stable apple juice: Integrating GC-MS fingerprinting and chemometrics. Foods, v. 9, p. 1-13, 2020. https://doi.org/10.3390/foods9020165.

KIST, B. B. et al. Anuário brasileiro da maçã 2019. Santa Cruz do Sul: Editora Gazeta Santa Cruz, 2019. 56 p.: il.

KOUTSOS, A. L. et al. Effects of commercial apple varieties on human gut microbiota composition and metabolic output using an in vitro colonic model. Nutrients, v.9, n.6, p. 533, 2017. https://doi.org/10.3390/nu9060533.

LARSSON, S.C.; VIRTAMO, J.; WOLK, A. Total and specific fruit and vegetable consumption and risk of stroke: A prospective study. Atherosclerosis, v. 227, n.1, p. 147-152, 2013. https://doi.org/10.1016/j.atherosclerosis.2012.12.022.

LEE, J. et al. Analysis of volatile compounds as quality indicators for Fuji apples after cold storage. Journal of Food Biochemistry, v. 41, p. e12410, 2017. https://doi.org/10.1111/jfbc.12410.

LEE, Y-Y; JEONG M-C; JANG, H. W. Determination of volatile compounds by headspace-solid phase microextraction-gas chromatography/mass spectrometry: quality evaluation of Fuji apple. Analytical Science \& Technology, v. 30, n. 2, p. 6874, 2017. https://doi.org/10.5806/AST.2017.30.2.68.

LI, D. et al. Young apple polyphenols postpone starch digestion in vitro and in vivo. $\begin{array}{lllll}\text { Journal of } & \text { Functional } & \text { Foods, v. }\end{array}$ https://doi.org/10.1016/j.jff.2019.03.009.

LÓPEZ, M. L. et al. Volatile compounds, quality parameters and consumer acceptance of 'Pink Lady1' apples stored in different conditions. Postharvest Biology and Technology. v. 43, p. 55-66, 2007. https://doi.org/10.1016/j.postharvbio.2006.07.009.

LUMPKIN, C. et al. 'Fuji' apple (Malus domestica Borkh.) volatile production during high pCO2 controlled atmosphere storage. Postharvest Biology and Technology, v.100, p.234-243, 2015. https://doi.org/10.1016/j.postharvbio.2014.10.008.

MATTHEIS, J.P. et al. Interactive responses of Gala apple fruit volatile production to controlled atmosphere storage and chemical inhibition of ethylene action. Journal of 
Agriculture and Food Chemistry. v.53, p.4510-4516, 2005. https://doi.org/10.1021/jf0501210.

MEDINA, S. et al. Differential volatile organic compounds signatures of apple juices from Madeira Island according to variety and geographical origin. Microchemical Journal, v. 150, p. 104094, 2019. https://doi.org/10.1016/j.microc.2019.104094.

MEIGH D. F.; HULME A. C. Fatty acid metabolism in the apple fruit during the respiration climacteric. Phytochemistry, v. 4, p. 863-871, 1965. https://doi.org/10.1016/S0031-9422(00)86264-0.

MEIGH D. F.; JONES J. D.; HULME, A. C. The respiration climacteric in the apple: production of ethylene and fatty acids in fruit attached to and detached from the tree. Phytochemistry. $\quad$ v. 6 , p.1507-1515, 1967. https://doi.org/10.1016/S00319422(00)82943-X.

NELSON, D.; COX, M. Lehninger principles of biochemistry. New York: W.H. Freeman \& Co, 2004. 1119p.

NIJSSEN, L. M.; VAN INGEN-VISSCHER, C. A.; DONDERS, J. J. H. VCF. Compostos voláteis em alimentos: banco de dados (Versão 13.1.). TNO Triskelion, Zeist (Holanda), 2011.

NIU, Y. et al. Characterization of ester odorants of apple juice by gas chromatographyolfactometry, quantitative measurements, odour threshold, aroma intensity and electronic nose. Food Research International, v. 120, p. 92-101, 2019. https://doi.org/10.1016/j.foodres.2019.01.064.

NOGUEROL-PATO, J. et al. Active odorants in Mouratón grapes from shoulders and tips into the bunch. Food Chemistry. v. 133, p. 1362-1372, 2012. https://doi.org/10.1016/j.foodchem.2012.01.113.

PAILLARD, N. Biosynthese de produits volatils de la pomme: Formation des alcools et des esters á partir des acides gras. Phytochemystry, v. 18, p. 1165 1171, 1979. https://doi.org/10.1016/0031-9422(79)80127-2.

PARLIMENT, T. H. Solvent extraction and distillation techniques. In. MARSILI, R. Techniques for analyzing food aroma. New York: Marcel Dekker Inc., 1997, p. 17-44.

PAWLISZYN, J. Handbook of solid phase microextraction. Beijing: Chemical Industry Press, 2009. 406p.

PERESTELO, R. et al. Differentiation of fresh and processed fruit juices using volatile $\begin{array}{llll}\text { composition. } \quad \text { Molecules. } & \text { v.24, } 2019 .\end{array}$ https://doi.org/10.3390/molecules24050974.

PÉREZ A. G.; SANZ, C. Formation of fruit flavour. In: BRÜCKNER, B.; WYLLIE, S. G., editors. Fruit and vegetable flavour. Boca Raton: CRC Press, 2008. p. 71-102.

POWER, F.B.; CHESTNUT, V.K. The odorous constituents of apples. Emanation of acetaldehyde from the ripe fruit Journal of the American Chemical Society. v. 42 p. 1509-1526, 1920. https://doi.org/10.1021/ja01452a029. 
PRANGE, R. K. Dynamic Controlled Atmosphere (DCA) storage of fruits and vegetables. In. Reference Module in Food Science (p. B9780081005965214000), 2018. https://doi.org/10.1016/B978-0-08-100596-5.21349-8.

QIN, Z.; PETERSEN, M. A.; BREDIE, W. L. P. Flavor profiling of apple ciders from the UK and Scandinavian region. Food Research International, v.105, p.713-723, 2018. https://doi.org/10.1016/j.foodres.2017.12.003.

REINECCIUS, G. Flavor chemistry and technology. Boca Raton: CRC Press, 2006. 520p.

RISTICEVIC et al. Recent developments in solid-phase microextraction. Analytical and Bioanalytical Chemistry, v.393, n.3, p. 781-795, 2009. https://doi.org/10.1007/s00216-008-2375-3.

ROWAN D. D. et al. Biosynthesis of straight-chain ester volatiles in Red Delicious and Granny Smith apples using deuterium-labeled precursors. Journal of Agricultural and Food Chemistry, v.47, p. 2553-2562, 1999. https://doi.org/10.1021/jf9809028.

SALAZAR, N. O., OROZCO, G. O. El aroma de la manzana. Interciencia, v.36, p. 265271, 2011.

SANZ, C.; OLÍAS J. M.; PÉREZ A. G. Aroma biochemistry of fruits and vegetables. In. TOMÁS BARBERÁN, F.A.; ROBINS, R.J., editors. Phytochemistry of fruits and vegetables. Oxford: Clarendon Press, 1997, p.125-255.

SAQUET, A. A.; BRACKMANN, A.; STORCK, L. Armazenamento de maçã Gala sob diferentes temperaturas e concentrações de oxigênio e gás carbônico. Ciência Rural, v.27, n.3, p.399-405, 1997. https://doi.org/10.1590/S0103-84781997000300006.

SAQUET, A. A.; STREIF, J. Fermentative metabolism in 'Jonagold' apples under controlled atmosphere storage. European Journal of Horticultural Science, v. 73, p. 43-46, 2008. https://doi.org/10.1590/1678-4499.189.

SCHMUTZER, G.R. et al. Determination of the volatile components of apple juice using solid phase microextraction and gas chromatography-mass spectrometry. Analytical Letters, v.47, p. 1683-1696, 2014. https://doi.org/10.1080/00032719.2014.886694.

SONG, J.; BANGERTH, F. Fatty acids as precursors for aroma volatile biosynthesis in pre-climacteric and climacteric apple fruit. Postharvest Biology and Technology. $v$. 30, p.113-21, 2003. https://doi.org/10.1016/S0925-5214(03)00098-X.

TAKEOKA, G. R; BUTTERY, R. G; FLASH, R. A. Volatile constituents of Asian pear (Pyrys serotina). Journal of Agricultural and Food Chemistry. v. 40, p.1925-1929, 1992. https://doi.org/10.1021/jf00022a040.

THEWES, F. R. et al. Dynamic controlled atmosphere storage suppresses metabolism and enhances volatile concentrations of 'Galaxy' apple harvested at three maturity stages. Postharvest Biology and Technology, v. 127, p. 1-13, 2017a. https://doi.org/10.1016/j.postharvbio.2017.01.002.

THEWES, F. R. et al. The different impacts of dynamic controlled atmosphere and controlled atmosphere storage in the quality attributes of 'Fuji Suprema' apples. 
Postharvest Biology and Technology, v.130, p.7-20. 2017b. https://doi.org/10.1016/j.postharvbio.2017.04.003.

VALERIO, F. et al. The viability of probiotic Lactobacillus paracasei IMPC2.1 coating on apple slices during dehydration and simulated gastro-intestinal digestion. Food Bioscience, v.34, p. 100533, 2020. https://doi.org/10.1016/j.fbio.2020.100533.

VARMING, C. et al. Comparison of isolation methods for the determination of important aroma compounds in Black Currant (Ribes nigrum L.) Juice, Usin Nasal Impact Frequency Profiling. Journal of Agricultural and Food Chemistry, v.53, n.6, p. 1647162, 2004. https://doi.org/10.1021/jf035133t.

VELTMAN, R. H.; VERSCHOOR, J. A.; RUIJSCH VAN DUGTEREN, J. H. Dynamic control system (DCS) for apples (Malus domestica Borkh. Cv. 'Elstar'): optimal quality through storage based on products response. Postharvest Biology and Technology, v. 27, p. 79-86, 2003. https://doi.org/10.1016/S0925-5214(02)00186-2.

WEBER, A. et al. Atmosfera controlada para o armazenamento da maçã 'Maxi Gala'. Revista Ciência Agronômica, v. 44, n.2, p. 294-301, 2013. https://doi.org/10.1590/S1806-66902013000200011.

WEBER, A. et al. Dynamic controlled atmosphere (DCA): interaction between DCA methods and 1-methylcyclopropene on 'Fuji Suprema' apple quality. Food Chemistry, v. 235, p.136-144, 2017. https://doi.org/10.1016/j.foodchem.2017.05.047.

WRIGHT, A. H.; ARUL, J.; PRANGE, R.K. The trend toward lower oxygen levels during apple (Malus domestica Borkh) storage - A review. Journal of Horticultural Science \& Biotechnology, v.90, p.1-13, 2015. https://doi.org/10.1080/14620316.2015.11513146.

WYLLIE, S.; FELLMAN J. K. Formation of volatile branched chain esters in bananas. Journal of Agricultural and Food Chemistry, v. 48, p. 3493-3496. 2000. https://doi.org/10.1021/jf0001841.

$\mathrm{XU}, \mathrm{C}-\mathrm{H}$. et al. Applications of solid-phase microextraction in food analysis. Trends in Analytical Chemistry, v. 80, p. 12-29, 2016. https://doi.org/10.1016/j.trac.2016.02.022.

$\mathrm{XU}$, J. et al. New materials in solid-phase microextraction. Trends in Analytical Chemistry, v.47, p. 68-83, 2013. https://doi.org/10.1016/j.trac.2013.02.012.

\section{Autores}

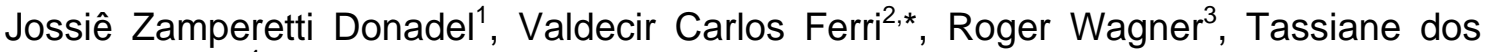
Santos Ferrão ${ }^{4}$

1. Curso de Pós-Graduação Lato Sensu em Ciência dos Alimentos, Centro de Ciências Químicas, Farmacêuticas e de Alimentos, Universidade Federal de Pelotas, Campus Capão do Leão, Caixa Postal 354, CEP. 96010-900, Pelotas, RS, Brasil; Centro de Ciências Rurais, Universidade Federal de Santa Maria, Campus Sede, CEP. 97105-900, Santa Maria, RS, Brasil. 
2. Centro de Ciências Químicas, Farmacêuticas e de Alimentos, Universidade Federal de Pelotas, Campus Capão do Leão, Caixa Postal 354, CEP. 96010-900, Pelotas, RS, Brasil.

3. Centro de Ciências Rurais, Universidade Federal de Santa Maria, Campus Sede, CEP. 97105-900, Santa Maria, RS, Brasil.

4. Instituto Federal de Educação, Ciência e Tecnologia de Roraima (IFRR), Campus Novo Paraíso, CEP. 69365-000, Caracaraí, RR, Brasil.

*Autor para correspondência: ferriufpel@gmail.com 